Davies, E, Jolley, D and Coiffait, F

Reflection and connection: UK Psychologists' views and experiences of blogging

Davies, E, Jolley, D and Coiffait, F (2017) Reflection and connection: UK Psychologists' views and experiences of blogging. ELearning and Digital Media

doi: $10.1177 / 2042753016689634$

This version is available: https://radar.brookes.ac.uk/radar/items/79c0d9e9-733e-4067-ab7c-06492780f2d5/1/

Available on RADAR: February 2017

Copyright $(C$ and Moral Rights are retained by the author(s) and/ or other copyright owners. A copy can be downloaded for personal non-commercial research or study, without prior permission or charge. This item cannot be reproduced or quoted extensively from without first obtaining permission in writing from the copyright holder(s). The content must not be changed in any way or sold commercially in any format or medium without the formal permission of the copyright holders.

This document is the postprint version of the journal article. Some differences between the published version and this version may remain and you are advised to consult the published version if you wish to cite from it. 


\section{Reflection and connection: UK Psychologists' views and experiences of blogging}

Emma L. Davies, Oxford Brookes University, UK.

Daniel Jolley, Staffordshire University, UK.

Fleur-Michelle Coiffait, Sheffield Children's NHS Foundation Trust, UK.

Corresponding author: Dr Emma L Davies edavies@brookes.ac.uk, Department of Psychology, Social Work \& Public Health, Oxford Brookes University, Headington Campus, Gipsy Lane, Oxford, OX3 0BP.

Keywords: blogging, academia, digital communication, psychologists,

Running header: Psychologists and blogging

Word count 6365 


\title{
Reflection and connection: UK Psychologists' views and experiences of blogging
}

\begin{abstract}
The value of using social media is being increasingly recognised among the academic community. Blogging has been identified by some researchers as a means of reaching a nonacademic audience and in order to increase research citations. However, there is little research exploring this method of communication within specific disciplines. This study, therefore, explored UK psychologists' views and experiences of blogging using an online survey, of both blog writers and non-blog writers. In addition, the study sought to determine whether those who identified as blog writers when they completed the survey, and who consented to a content analysis of their posts, were still posting regularly 12 months later. The majority of the blog writers said that they wrote blogs as a means of expressing themselves and their research. Content analysis reflected the survey findings, with research as the most common category for posts, but we identified that the intended audience for such posts appeared to be other academics. Interestingly, 37\% of blog writers had stopped regularly posting at the point where posts were analysed, 12 months after the survey. Moreover, despite positive attitudes and subjective norms about blogging across the sample, we also identified that for respondents who were non-blog writers were reluctant to blog because they worried that their contribution may not be valued, or that they did not have the time. These findings highlight that there is work to do in order to convince psychologists to engage - and to continue doing so - with this medium of communication.
\end{abstract}




\section{Reflection and connection: UK Psychologists' views and experiences of blogging}

The value of having a social media presence, such as a twitter account or a blog, is being increasingly recognised by the academic community (Mewburn \& Thomson, 2013; Meyer \& McNeal, 2011; Veletsianos \& Kimmons, 2016). It has been suggested that social media use can both enhance research impact, and contribute to greater transparency in the research process (Veletsianos \& Kimmons, 2012). To this end, a small number of studies have explored academics' use of social media, aiming to describe its use and quantify the benefits. The current paper focusses on the use of blogs, which are websites where an individual or team of individuals post regular timed updates, akin to diary entries, appearing in reverse chronological order. While blogs are used by a wide range of academics and students from all disciplines, here, we explore how blogs are used and viewed by psychology academics and students.

Blogs are used in a wide variety of ways; their flexibility and reach can allow anyone to self-publish on a topic of their choice (Ewins, 2005). Blogs are also different from other social media as they involve communication with a wider audience, often people that the writer does not know, in comparison to the 'anchored' communication with 'friends' offered by social networks such as Facebook (Fullwood, Melrose, Morris, \& Floyd, 2013). Thus, the practice of blogging could be seen to fall between the ethos of print media and social networking. Indeed, both the speed and ease of communication mean that blogs have advantages over traditional media; for example, they are published instantly and they allow a dialogue between the writer and the reader (Shema, Bar-Ilan, \& Thelwall, 2012). A number of academics from a wide range of disciplines have been quick to exploit the benefits of blogging, seeing the potential to participate in an international network of those interested in the same research areas (Stafford \& Bell, 2012). However, despite the range of potential benefits, blogging is not widespread in academia (see Green, 2015 for a brief discussion). 
One advantage of writing a blog as an academic is the potential to reach a wider audience than the traditional journal publication, which may be concealed behind a paywall. Stafford and Bell (2012) suggest that the use of blogging in academia therefore allows nonacademics to comment on and share ideas on current research and debates, potentially increasing impact. With more focus on research impact, it is unsurprising that those academics who blog have sought to demonstrate their reach through this medium. The London School of Economics (LSE) blog is one example of a group of academics joining together to communicate about important research findings. LSE also produced a recent guide for academics who use Twitter, which has been widely disseminated (see Mollet, Moran, \& Dunleavy, 2011). The LSE has now produced an academics' Twitter guide, demonstrating an increased recognition of the importance of engaging in online communities of this nature.

Blogs may also be used by $\mathrm{PhD}$ students to develop their academic identity and reach out to a wider network in order to improve the writer's future prospects (Bennett \& Folley, 2014; Ewins, 2005). Recently, Bennett and Folley (2014) reflected on using blogs during their $\mathrm{PhD}$ studies. They discussed the development of doctoral student identity and how $\mathrm{PhD}$ students learn to 'enact scholarship' and cope with a dual identity as student and staff member at the time. Their use of blogging was a positive experience, enabling them to deal with some of the challenges of $\mathrm{PhD}$ life, and creating an effective writing habit. Ewins (2005) calls for a cautious approach, however, when reflecting on his own blogging experiences. He argues that a blogger may not be successful in a job application in comparison to a non-blogger, for fear of being seen as a 'dissenter' or someone who airs the department's dirty laundry (Ewins, 2005; Tribble, 2005).

Blogs are also used in teaching, both in traditional face-to-face brick institutions and as a means for distance learners to communicate with peers. For example, one study found 
that using blogs helped to create a sense of community and collaborative learning among student teachers, although this did depend on individual computer knowledge (Top, 2012). Another study found that increasing sense of community through blogging led to a better learning experience for students (Hanuscin, Cheng, Rebello, Sinha, \& Muslu, 2014). In a further study, blogging was applied in a large lecture class, where students can typically feel disconnected. The researchers found that the use of blogs helped foster a sense of community and helped the students' learning; with the blogging tasks helping them to share knowledge with one another (Halic, Lee, Paulus, \& Spence, 2010).

In order to understand some of the uses and benefits of blogging for academics, Mewburn and Thompson (2013) analysed the content of 100 academic blogs. They identified seven key themes within their content analysis: self-help, academic practice, technical advice, academic culture critique, research dissemination, career advice, and personal reflections. They also examined the intended audience of each blog, highlighting the difference between those meant for other academics and those aimed at public engagement. Mewburn and Thompson described a kind of 'virtual staff room' where ideas could be shared, discussed and critiqued. They concluded that blogging should be considered as a "social community of practice in context of current institutional pressures and higher educational policy" (Mewburn \& Thompson, 2013, p. 1118).

A more recent content analysis examined blogs specific to health research from Researchblogging.org (Shema, Bar-Ilan, \& Thelwall, 2015). Due to discussions regarding the use of alternative citation metrics, the focus of their study was to explore how peer reviewed references were used within a sample of blogs. The authors found that most academic bloggers used conventional references, perhaps as a persuasive tool, and that these were general references to very recent publications. Interestingly, they also found that only a small number of bloggers cited their own research. The purpose of blogging about a 
published piece appeared to be in order to provide some discussion of a paper, sometimes offering a critique or suggesting an extension to the research (Shema et al., 2015).

Although much research on the nature and purpose of academic blogging has emerged over the last few years, there are fewer studies that focus on the use of blogging within specific disciplines. As highlighted by Shema et al., (2015), we do need to gain an understanding of the motivations for specific research disciplines. Research exploring these questions is, therefore, timely. Whilst the specific benefits of engaging in blogging for psychologists have not been explored prior to this investigation, several popular Psychology blogs exist. Shema et al. (2012) also found that Psychology was a popular category for blog posts. A specific example comes from the British Psychological Society (BPS), who run the Research Digest blog, which aims to summarise new and interesting published research for the general public. Managed by Dr Christian Jarrett with contributions from other bloggers, it attracts 337,000 readers each month (Research Digest, 2015). Stafford and Bell (2012) suggested that social media competence is no longer optional for psychologists, even though some may still doubt its value. As Mewburn and Shiner (2013) highlight, despite the many benefits, academic blogging is inevitably an 'extra-curricular' activity. Understanding why some psychologists' blog and others do not is thus an important avenue for exploration. In addition, while there is some existing research from other academic disciplines, professional psychologists may work as academics or practitioners, and it appears that little is known about practitioners' use of blogs.

Another reason why a focus on blogging is timely is that a study has shown that blogging about research also has the potential to lead to higher rates of citations for papers (Thelwall, Haustein, Larivière, \& Sugimoto, 2013). In addition, as highlighted by Shema et al. (2012), blogging may be used as part of academics' research evaluation. Thus, an exploration of blogging by psychologists is warranted due to the potential for blog writing to 
contribute to important metrics that academics are judged upon, such as article downloads and citation counts. Veletsianos and Kimmons (2013) highlighted that academics may feel under too much time pressure to engage with online social networks and so it is possible that some people may start to write a blog, and then find that they do not have the time to maintain it. Therefore, one further point of interest relates to whether would be bloggers can maintain a blog. Do those who intend to maintain a blog really last the distance when it comes to producing a blog in the long term? A useful framework for exploring the relationship between intentions and behaviour within Psychology is the Theory of Planned Behaviour (TPB) (Ajzen, 1991). Bloggers and current non-bloggers may both have high levels of intention to blog but may find that time pressures reduce their actual blogging behaviours, either in the frequency of posts or in not starting a blog. Attitudes, social norms, and perceived control are important for forming intentions to engage in behaviour (Ajzen, 1991). Thus it may be informative to explore these constructs in relation to blogging in order to provide additional explanations for blogging behaviours amongst psychologists.

Thus, the current survey was conducted in order to explore psychologists' use of and views about blogging. Our primary aims were to find out what motivated current blog writers to engage with this medium (by exploring their attitudes, intentions, subjective norms and perceived control over blogging), and to explore the topics that these bloggers were writing about. In addition, we also sought to determine whether those who identified as blog writers when they completed the survey, and who consented to a content analysis of their posts, were still posting regularly 12 months later. Our secondary aim was to find out what psychologists who were not currently writing a blog themselves, thought about the medium of blogging and its purposes by exploring their attitudes, intentions, subjective norms and perceived control over blogging. 


\section{Method}

\section{Participants}

The target group of participants for this survey were psychologists at any stage in their career. Recruitment advertisements were placed online using social media and a letter was printed in The Psychologist explaining the purpose of the project and inviting people to take part. Seventy-six respondents aged 19-73 (Mean age $=34)$ completed the survey $(63 \%$ female). The largest group of respondents identified as postgraduate students $(n=30$; $39.5 \%)$, followed by academics $(n=21 ; 27.6 \%)$. There were 14 undergraduate students $(18.4 \%)$ and 11 participants who identified as practitioners $(14.5 \%)$.

Of the 76 participants, 49 (64\%) described themselves as current bloggers with 66 (87\%) also reporting that they were blog readers. The 49 current blog writers age ranged 20 73 (Mean age $=32.96 ; 77.8 \%$ female) and the 27 non-blog writers age ranged 20-73 (Mean age $=34.39 ; 55 \%$ female $)$. Of the current blog writers $(n=49), 11$ per cent had been blogging for less than 6 months and 12 per cent for more than five years.

\section{Procedure}

The survey was developed by XX and XX and piloted with two psychologists with an interest in this topic prior to data collection taking place. The survey was collected using a Google Docs survey with the first page comprising a participant information sheet. Participants were told that consent was implied by completion of the survey, but that they were free to withdraw before submitting their responses. Ethical approval for the study was obtained from XXXX University Research Ethics Committee (study reference number 130744). 


\section{Measures}

The survey had three sections. The first section was about blogging experience and practice. The second section was on views about blogging. The third section asked participants for demographic details about themselves.

\section{Blogging experience and practice}

In this section, the survey sought to examine the prevalence of blogging and engagement with other blogs. We asked participants whether they were current bloggers and their reasons for doing so (or not). We asked those who blogged how long they had been maintaining their blog and what topics they wrote about (Figure 1). Within this section, we were also interested in what blogs our participants regularly read, and the topics that they were interested in reading about. We also asked them about their reasons for reading blogs and to provide a list of those they regularly visited.

\section{Views about blogging}

In this section, we asked participants about what they thought were the main purposes of blogging, asking them to select from a list (see Figure 2) and to provide their own. We also measured views about blogging using 9 statements and asking participants to rate their agreement from 1 (strongly disagree) to 7 (strongly agree). For example, whether blogging was a good use of time and the importance of being careful what is posted online (see Table 1 for all statements). Five statements $(3,4,6,7$, and 8$)$ were reverse scored so that a higher score for each statement indicated a positive view of blogging. The items were summed into a 'blogging views' scale $(\alpha=.74)$.

To further explore whether psychologists who held positive views of blogging held intentions to blog themselves, we asked participants to indicate their intention to blog on a 
scale from 1 (very unlikely) to 7 (very likely). Participants were asked about their attitudes towards blogging once a month for the next year on two seven-point bi-polar scales (goodbad and unpleasant-pleasant; the first item was reverse scored and the two item scale had good reliability $\alpha=.88$ ). We also asked participants to indicate the subjective norms (phrased as 'most people who are important to me would approve of my blogging'), on a scale from 1 (strongly disagree) to 7 (strongly agree) and their perceived control to post blogs (phrased as 'I am confident that I can blog once a month for the next year), from 1 (strongly disagree) to 7 (strongly agree). We included such measures as they are regularly measured as a standard format for TPB questions (Francis et al., 2004). At the end of this section, we also asked an open question to seek information about whether there were things that participants wanted to find out or learn about blogging.

\section{Demographics}

In this final section, we asked participants for their age, gender and their current role or job position. We asked for the blog URL of those who were maintaining a blog and were willing to share this, and sought permission to include these blogs in the subsequent content analysis.

\section{Results}

There was no significant difference in the ages of bloggers versus non-bloggers. This was, therefore, not included in the following analyses.

Blog writers $(n=49)$

There was large variability in terms of the frequency of posts by current blog writers, with the most common response being once a month. Forty-seven percent reported blogging about work topics, 22 per cent blogged about social life or non-work interests and 31 percent said 
they blogged about both work and outside interests. The main reasons for blogging $(n=18)$ were about reflection and communication about research, as exemplified in the following comments:

"Reflect on and track my progress, to make sense of things, or enjoyment" (P4; postgraduate)

"As a way to tell people about my research" (P33; Academic)

A need to get things out in the open, or respond to news or research papers was another common reason $(n=8)$, as another participant elaborated:

"Frustration, need to express" (P69; Academic)

Non-blog Writers $(n=27)$

For non-blog writers, however, time $(n=12)$ was highlighted as one of the obstacles to not writing their own blog:

Not enough time, worry I haven't got anything to say! (P5; postgraduate)

Not enough time, not sure what content would be, find blogging a bit self-obsessional and indulgent. (P42; practitioner)

Others suggested that they were worried that they did not have anything interesting to say, or would not be perceived as authoritative $(n=7)$.

Who would want to read my ramblings anyway (P12; postgraduate)

Another non-blog writer discussed their preference for gaining information from more established sources: 
I prefer to get information on topics of interest from professional or academic sources (P6; undergraduate)

In summary blog writers said they saw the medium as a positive way to engage with other people, but non-blog writers saw time or a lack or

Why read a blog?

Fifty five percent of blog readers had been doing so for between 3-4 years and 39\% for more than five years. Thirty six percent reported reading blogs daily. Most of the participants read blogs about both work and outside interests (68\%). Fifteen per cent read blogs solely for work reasons and 17 per cent for social life or non-work interests. The specific content of the blogs that readers were engaged in are shown in Figure 1. Participants were also asked to report the names of the blogs that they frequently read. The British Psychological Society (BPS) Research Digest http://digest.bps.org.uk/, Bad Science, http://www.badscience.net/ and Bishop Blog http://deevybee.blogspot.co.uk/ were the most frequently cited; however a very wide range (76 different blog sources) were mentioned.

\section{[Insert figure 1]}

The main reasons for reading blogs were to engage with topics of interest $(n=24)$ keep up to date $(n=12)$ and to connect with other people experiencing the same issues $(n=$ 8), as elaborated by the two participants below:

"Information of topics of interest, to gain insight into the lives of people who are dealing with the same issues and difficulties in life" (P29; undergraduate)

"Keep up to date with other research, events and funding. To keep up to date with some social stuff" (P63; Postgraduate) 


\section{Attitudes towards blogging}

Within the 'views about blogging' scale participants across the sample reported that sharing personal reflections and thoughts was the main purpose of blogging $(n=65)$, closely followed by the dissemination of information $(n=54)$. A smaller number $(n=18)$ saw the purpose of blogging to be to further their career. Other respondents added that they wanted to market their business, practice amateur journalism and keep a record of articles (Figure 2).

\section{[Insert figure 2]}

Overall, there were high levels of agreement that one should take care what one posted online (see Table 1) and that some topics were not appropriate. Furthermore, participants tended to agree that blogging was a good use of time and that $\mathrm{PhD}$ students should blog. Participants tended to disagree with the statement that blogging was a waste of time, and there was a tendency to disagree with statements about not having time, and that blogging would not enhance someone's career (Table 1).

\section{[Insert table 1]}

We also asked about norms, perceived control and intentions to blog (see Table 2). Subjective norms about blogging were generally positive, suggesting that respondents thought blogging was approved of by other people. There was a large variation in perceived control to blog and intentions to blog fell in the middle of the scale with the large variation in responses possibly reflecting that some respondents were regular bloggers whereas others were not. Spearman's Rho correlations were calculated to examine the relationship between these measures and the blogging views scale across the whole sample. Blogging attitudes were positively correlated with subjective norms $(r(76)=.311, p=.006)$, perceived control $(r(76)=.483, p<.001)$ and intentions to blog $(r(76)=.58, p<.001)$. There was, however, no 
relationship found with attitudes towards blogging once a month for the next 12 months $(p>$. 05). Holding a positive view of blogging was therefore related to perceived norms of the social group, giving a sense of control and also being more likely to blog.

Next, we then compared blogging views, attitudes, subjective norms, perceived control and intentions between bloggers $(n=49)$ and non-bloggers $(n=27)$ (see Table 2). There were significant differences between the two groups in terms of intentions to blog, perceived control and in the general blogging views scale. Bloggers had greater intentions to blog had more positive general views and had a greater sense of perceived control over blogging. Attitudes and subjective norms were similarly high, showing that both groups had positive attitudes towards blogging and believed it to be something that would be approved of by others.

[Insert table 2]

We also compared these measures between the participant groups (see Table 3). While there were no significant differences in the measures, some interesting patterns can be observed. For example, practitioners have lower perceived control and lower subjective norms about blogging compared to the other groups. Academics have higher intentions and higher perceived control than the other groups.

[Insert table 3]

\section{Content analysis}

Forty-five blogging participants provided their blog URL and consented for content analysis to be undertaken on their blogs. The content analyses provided the opportunity to assess the topics bloggers wrote about, alongside whether participants were still blogging after 12 months. All 45 blogs were examined and included in the content analysis if they had 
posted a total of five separate posts with at least one in the last six months (as of $29^{\text {th }}$ August 2014). This left a total of 28 blogs (20 identified as written by academics, four by practitioners and 4 by others, including media commentators), for which the five most recent posts were coded by $\mathrm{XX}, \mathrm{XX}$ and a research assistant (XX). We conducted a quantitative content analysis where we coded manifest categories, with individual posts as the unit of analysis. We undertook the analysis using the seven steps outlined by Treadwell and used a similar process to Mewburn and Thompson (2013) to identify the categories of blog posts. An initial set of codes and themes was developed in discussions between XX and XX and a coding frame was developed (140 posts). Reliability between the three coders was high (88\% after initial coding). Disagreements were resolved in discussions between all coders until a final code for each post was agreed.

\section{[Insert table 4]}

\section{Research:}

The most common code applied to the blog posts was related to research. This broad category represents posts that relate to either the bloggers' own research (58\%) or their commentary or reflection on other published research. For example, some bloggers published a post when they had a new peer reviewed publication accepted in a traditional journal. However, this category also included posts about research and publishing in general. For example, bloggers have written about trust in scientific publishing in response to debates about falsified findings. This map wells on to the responses provided by blog writers on the purposes of their blog.

\section{Advice:}


The advice category was the only place where we identified practitioners openly blogging, in comparison to academic bloggers who were present in all of the other categories. Here, advice was for potential clients on their conditions and other sources of support. For example, on one practitioner's blog, the author posted about dealing with stress during the festive period. This type of blog may serve to help general readers, as well as attract new clients to the business. Although practitioners had lower levels of perceived control and intentions to blog, perhaps giving advice and support is one area that they could be encouraged to contribute. Other advice posts were related to the $\mathrm{PhD}$, careers, or statistical techniques. Another blogger wrote about their top tips for surviving a clinical psychology $\mathrm{PhD}$.

Public engagement:

Blog posts were coded as relating to public engagement where their intended audience was those outside of academia. For example, Brain Flapping hosted by The Guardian, uses a humorous style to dissect science stories that have been reported in the media. An example that we noted was from January where the blog author attempts to dispel the often heard myth that the second Monday in January is the "most depressing day of the year'. Similarly, the blog Mindhacks also reported on diverse topics in an accessible way. This finding chimes survey findings suggesting that participants see blogging as a way to engage with new audiences.

Teaching:

A small number of bloggers were writing posts related to their teaching of Psychology. For example, posting on the topic of plagiarism in students and arguing that students are often treated as though they are continuously trying to cheat and that plagiarism was a moral panic. Another blogger included posts on his dissertations students' project 
work, requesting readers to take part in their online studies. This may be one way that bloggers reach new audiences as well as vent frustrations.

Response:

The 'response' category was created to reflect that blog posts had been used to counter a story in the media, or reflect on a public event. To turn to Brain Flapping once more, a post was written relating to the media's reporting of the actor Robin Williams' apparent suicide. The blog author was critical of the mainstream media reporting of the method of suicide and of those who proclaim suicide to be a selfish act. Here, the relative speed of publication of this blog post was of upmost importance following other media coverage of the actor's death. This category relates to the survey finding that sharing personal reflections and thoughts was a key purpose of blogging for the respondents.

\section{PhD Journeys:}

Some posts were written by students in the throes of their PhD studies, giving advice or reflecting on the experience. PhD students may be under increasing pressure to add value and set themselves apart from their contemporaries in a competitive job market. The the posts we coded in this section were reflections on the $\mathrm{PhD}$ experience rather than critiques of research or commentary. This category may reflect the large number of postgraduate students in our sample. It may also reflect the survey finding that development of writing skills and furthering careers was a key purpose of blogging for the participants.

\section{Conferences:}

A smaller number of blog posts were coded as being related to conferences. Two of these were reviews of conference talks by $\mathrm{PhD}$ students, whereas the others were more general comments about conferences that the writer had attended. For example, one blogger 
wrote about a keynote talk which had inspired him to think about public engagement. This may also reflect the large number of postgraduate participants, who may be looking for topics on which to communicate with a wide audience during the early stages of their career.

\section{Other interests:}

Our 'other interests' category reflects a vast array of non-Psychology content. For example, our bloggers were posting their recipes, reflections on parenthood, descriptions of places in the UK, and hobbies such as knitting. The large number of posts in this category may reflect a need for an outlet to express other ideas and interests for those engaged in work related to Psychology. Interestingly, many of these blogs were anonymous, in contrast with those in the research category. This is an interesting finding that suggests that connecting with other academics and the public was not a primary concern for those who maintained their blog in the long term, despite survey findings suggesting otherwise.

The participants who took part in our survey were therefore shown to blog about both research and non-research activities. In addition to examining the content of the blog posts, we sought to determine the writer's intended audience for their posts. While most blog posts that we read as part of this analysis were written in a non-academic, accessible style, the intended audience was often identified to be other academics or researchers.

\section{Discussion}

This study was conducted to explore psychologists' use of and views about blogging. Firstly, to identify what motivates current bloggers to write and to explore their attitudes, intentions, subjective norms and perceived control over blogging. We also sought to find out what bloggers were writing about and whether these blogs were maintained 12 months 
following the survey. In addition, as a secondary aim, we included non-bloggers in the sample to seek their views on the topic.

First, we consider the findings related to views about blogging from both the bloggers and non-bloggers. We found that bloggers reported doing so predominantly for work purposes, as a means of expressing themselves and their research. The majority of those who read blogs also do so for both research and non-research related purposes. The majority of respondents reported sharing personal reflections and thoughts as a primary purpose of blogging, with only 18 (out of 76) reporting that it was to further their career. Participants highlighted reflection and a need to respond to news or research as reasons for blogging. A lack of time, and a view that they were perhaps not authoritative sources was highlighted as reasons not to blog. Although our respondents were mainly positive about blogging (likely to be due to the predominance of bloggers within our sample), there was a high agreement to the statements in the survey about being careful what was posted online, and that some topics were not appropriate for blogging.

We found that participants' blogging views were associated with subjective norms, perceived control, and intentions to blog. Subjective norms about blogging were high, suggesting that perceived approval of this activity was positive. Perceived control over blogging was similar in bloggers and non-bloggers, suggesting that non-bloggers felt they could write a blog if they wanted to. However perceived control was lower for practitioners compared to the other groups. This might suggest a reluctance to post in case patients viewed posts, or that it might not be approved of by employers. Further research is needed to understand practitioners' views as our sample was relatively small.

We found that bloggers had greater intentions to blog had more positive general views and had a greater sense of perceived control over blogging. However, our blogging views 
scale was not associated with attitudes towards blogging once a month for the next 12 months. This could be because although participants held positive views overall about blogging, the commitment to maintain a regular blog might be influenced by other factors, such as time. Although participants appeared positive attitudes to blogs using the quantitative measures, the qualitative findings suggested concerns about what to write, having time to write and the authority of blogs may be barriers to actually starting or maintaining a blog.

Our content analysis allowed the categorisation of posts into topics and we additionally noted who the intended audience might be. In general, we noted that while the blog posts that we read as part of this analysis were written in a non-academic, accessible style, the intended audience was often identified to be other academics or researchers. The reasons provided for blogging concerned communication about research and discussing topics of interest. This supports Mewburn and Thompson's (2013) idea that blogs function as a kind of 'virtual staff room'. Similarly, within the content analysis of the blogs in our sample, we identified that research discussion was the most frequently coded topic. These findings suggest that most bloggers were not writing to engage the public with their research, despite Stafford and Bell's (2012) assertion that this was a key purpose of blogging. Furthermore, with only $58 \%$ of the research category related to the participants' own research, this suggests that most respondents were not blogging to increase citations of their papers.

Our 'other' category was the second most commonly coded within our sample of blog posts. This demonstrates the range and breadth of topics that Psychology bloggers were posting about and shows that many of the bloggers were writing about their personal and social lives. This is in contrast to Shema et al's (2015) findings, which suggest that health research bloggers appear to have a genuine desire to engage with a non-academic audience. 
A further aim of the study was to determine if bloggers who had consented to the content analysis were still posting 12 months later. We noted that of that of the 45 participants who provided their blog URL in the survey, only 28 were still blogging at 12 months. This may reflect an initial enthusiasm or commitment to blogging, which is unsustainable for many would-be bloggers. The time commitment cited by participants as a reason not to blog may have been an obstacle for these bloggers.

Green (2015) counteracts time as a barrier in a recent LSE post, who suggested that the writer should not spend that long composing their post, otherwise it may be inaccessible. Acknowledging that time is often an issue, Fullick (2011) gave an overview of the advantages and disadvantages of spending time on this activity. She suggested that many academics feel that blogging lacks legitimacy, in line with some of our participants' comments about preferring to get information from traditional sources and finding blogging self-obsessional and indulgent. We did find that a large proportion $(25.7 \%)$ of blog posts related to participants' social and personal interests rather than their professional activities. This is interesting given our findings related to the participants' views about blogging as beneficial, perhaps for some participants, it is an outlet for their interests away from work, rather than seen as a tool to engage with the public. It also suggests that traditional sources, such as journals, are still the best place to find legitimate information. One the issue of time, however, Fullick concluded that the potential for greater public engagement outweighed the drawbacks. Although a smaller number of our participants reported blogging to further their career, Fullick also argues that there is an increased recognition of the networking and career opportunities available to those who blog (Fullick, 2011).

Our results, therefore, highlight differences between the quantitative and qualitative survey findings, and the content analysis. While respondents had positive attitudes towards blogging and thought it was a good use of time, with some potential benefits, only a small 
proportion was still blogging 12 months following the survey. In addition, many of these were not blogging professionally, despite a generally high level of social approval for blogging and comments that suggested it was a good way to engage with the public. As these findings are somewhat contradictory, it would be beneficial to explore a wider sample of psychology-related blogs and to interview their writers to allow a more in-depth understanding of their motivations.

As with any piece of research, there are limitations that should be considered when reviewing our work. Our survey was completed by a self-selecting sample, notably comprising more active bloggers than inactive. The sample is also predominantly composed of students, and only a small proportion identified as practitioners. As this is a potentially interesting group, who may face different challenges to students and academics (for example around client confidentiality) it would be useful to conduct further research with this population. As one author points out, the use of social media by healthcare practitioners may be beneficial in enhancing openness between patients and providers and may help patients to access reliable information (Bacigalupe, 2011), and this benefit may also apply to clinical and other practising psychologists.

Although no claims for the generalisability of the findings are made, it would be advisable to seek a greater proportion of academics, practitioners and non-bloggers in a future study. Furthermore, we noted that many of the active bloggers who consented to the content analysis had ceased their practice by the time that the coding took place. This may reflect that an initial enthusiasm for blogging had been overridden by time and commitment factors. These findings are of course, only representative of UK psychologists. Whilst this may be a limitation, we do feel our findings could also help provide an understanding of blogging in other disciplines. 
Alongside future studies that seek to overcome the limitations discussed, these findings open up a range of future research directions. It appears that that psychologists view blogging for research as a good way to engage with the wider community, but that many of them are not using blogs in this way and blogs appear to be written with an academic audience in mind. This contradictory finding is interesting because it reveals that our respondents' beliefs and behaviours are not in line. It may be useful to explore what the bloggers themselves think about their intended audience, and what impact their think their blog is having.

However, little is really known about the 'impact' of blogging. It may, therefore, be useful to get a sense of policy makers' thoughts and experiences of blogging, and whether they actively engage in following blogs about relevant research topics. This is important due to the UK Research Excellence Framework's focus on research impact, which can be demonstrated by changes to policy and practice in the wider community. A further direction could examine the darker side of blogging and add to the literature examining trolling online. Indeed, a concern raised by the participants in our sample was the negative comments they do/may receive on their posts.

Another avenue could investigate the personal and career benefits of those people who post regularly on blogs. From this investigation, we have an understanding of why someone may blog (or not), but little is known about the social psychological consequences of blogging. These may be positive, such as providing a creative outlet, but may also be negative consequences due to the negativity that one may receive when blogging. A final avenue may investigate people's intentions and blogging. The content analysis included 45 blogs that were initially examined by the researcher; however, only 28 were found to have posted in the last six months, meeting criteria for inclusion in the analysis. This suggests that whilst there is a relationship between blogging views and intentions to blog, intentions may 
not lead to actual sustained blogging practices. Future research could further investigate this link.

\section{Conclusion}

Our study has provided as snapshot of UK psychologists' views and experiences about blogging. This timely study demonstrated some mixed views about the benefits of writing a blog; blogging can provide a channel to discuss research and open up about current issues, but it may be a heavy time commitment and not be a reliable source of information. We found that people's views on blogging were associated with social norms, perceived control and also intentions to blog. It may be important therefore to highlight the public engagement benefits to those who want to blog, but feel that time is a limiting factor.

\section{Acknowledgements}

The authors would like to thank the participants who completed the survey, in particular, those who consented to their blog posts being included in the content analysis. We are also grateful to XXXX for help with coding the content of the blogs, as well as to The Psychologist magazine for publishing our letter requesting participants to complete the survey. 


\section{References}

Ajzen, I. (1991). The theory of planned behavior. Organizational Behavior and Human Decision Processes, 50(2), 179-211.

Bacigalupe, G. (2011). Is There a Role for Social Technologies in Collaborative Healthcare? Families Systems \& Health, 29(1), 1-14. doi:10.1037/a0022093

Bennett, L., \& Folley, S. (2014). A tale of two doctoral students: social media tools and hybridised identities. Research in Learning Technology, 22, 23791. doi: http://dx.doi.org/10.3402/rlt.v22.23791

Ewins, R. (2005). Who Are You? Weblogs and Academic Identity. E-Learning, 2(4), 368377.

Francis, J., Eccles, M., Johnston, M., Walker, A., Grimshaw, J., Foy, R., . . Bonetti, D. (2004). Theory of Planned Behaviour Questionnaires: Manual for Researchers. Newcastle: Centre for Health Services Research, University of Newcastle.

Fullick, M. (2011). Should you enter the academic blogosphere? A discussion on whether scholars should take the time to write a blog about their work. Retrieved from http://blogs.lse.ac.uk/impactofsocialsciences/2011/11/30/should-you-enter-theacademic-blogosphere/

Fullwood, C., Melrose, K., Morris, N., \& Floyd, S. (2013). Sex, blogs, and baring your soul: Factors influencing UK blogging strategies. Journal of the American Society for Information Science and Technology, 64(2), 345-355. doi: 10.1002/asi.22736

Green, D. (2015). An antidote to futility: Why academics (and students) should take blogging / social media seriously. Retrieved from http://blogs.lse.ac.uk/impactofsocialsciences/2015/10/26/why-academics-andstudents-should-take-blogging-social-media-seriously/ 
Halic, O., Lee, D., Paulus, T., \& Spence, M. (2010). To blog or not to blog: Student perceptions of blog effectiveness for learning in a college-level course. The Internet and Higher Education, 13(4), 206-213.

doi: http://dx.doi.org/10.1016/j.iheduc.2010.04.001

Hanuscin, D. L., Cheng, Y. W., Rebello, C., Sinha, S., \& Muslu, N. (2014). The Affordances of Blogging As a Practice to Support Ninth-Grade Science Teachers' Identity Development as Leaders. [Article]. Journal of Teacher Education, 65(3), 207-222. doi: $10.1177 / 0022487113519475$

Mewburn, I., \& Thomson, P. (2013). Why do academics blog? An analysis of audiences, purposes and challenges. [Article]. Studies in Higher Education, 38(8), 1105-1119. doi: $10.1080 / 03075079.2013 .835624$

Meyer, K. A., \& McNeal, L. (2011). Academics online: Their interests and foibles. The Internet and Higher Education, 14(2), 113-120. doi: http://dx.doi.org/10.1016/j.iheduc.2010.09.002

Mollett, A., Moran, D., \& Dunleavy, P. (2011). Using Twitter in university research, teaching and impact activities Retrieved 15 December, 2015, from http://blogs.lse.ac.uk/impactofsocialsciences/files/2011/11/PublishedTwitter_Guide_Sept_2011.pdf

Shema, H., Bar-Ilan, J., \& Thelwall, M. (2012). Research Blogs and the Discussion of Scholarly Information. Plos One, 7(5), e35869. doi: 10.1371/journal.pone.0035869

Shema, H., Bar-Ilan, J., \& Thelwall, M. (2015). How Is Research Blogged? A Content Analysis Approach. Journal of the Association for Information Science and Technology, 66(6), 1136-1149. doi: 10.1002/asi.23239

Stafford, T., \& Bell, V. (2012). Brain network: social media and the cognitive scientist. Trends in Cognitive Sciences, 16(10), 489-490. doi: 10.1016/j.tics.2012.08.001 
Thelwall, M., Haustein, S., Larivière, V., \& Sugimoto, C. R. (2013). Do Altmetrics Work? Twitter and Ten Other Social Web Services. PLoS ONE, 8(5), e64841. doi: 10.1371/journal.pone.0064841

Top, E. (2012). Blogging as a social medium in undergraduate courses: Sense of community best predictor of perceived learning. The Internet and Higher Education, 15(1), 24-28. doi: http://dx.doi.org/10.1016/j.iheduc.2011.02.001

Tribble, I. (2005) Bloggers Need Not Apply, Chronicle of Higher Education, 8 July2015 Available at:

http://chronicle.com/jobs/2005/07/2005070801c.htm

Veletsianos, G., \& Kimmons, R. (2012). Assumptions and Challenges of Open Scholarship. International Review of Research in Open and Distance Learning, 13(4), 166-189.

Veletsianos, G., \& Kimmons, R. (2016). Scholars in an increasingly open and digital world: How do education professors and students use Twitter? The Internet and Higher Education, 30, 1-10. doi: http://dx.doi.org/10.1016/j.iheduc.2016.02.002 
Table 1 Median and interquartile range (IQR) of response to rating scale questions blogging views

\begin{tabular}{|c|c|c|}
\hline Statement & Median & $I Q R$ \\
\hline 1. Blogging is a good use of my time & 5 & 2 \\
\hline 2. I think that PhD students should blog & 5 & 2 \\
\hline 3. I do not have time to write a blog & 3 & 4 \\
\hline 4. Blogging would not enhance my career & 3 & 3 \\
\hline 5. It is important to be careful what you post online & 7 & 1 \\
\hline 6. There are some topics that are not appropriate for blogging & 6 & 4 \\
\hline 7. Blogging is a waste of time & 2 & 2 \\
\hline 8. There are more important things for me to do that write & 5 & 3 \\
\hline \multicolumn{3}{|l|}{ blogs } \\
\hline 9.I enjoy reading other people's blogs & 5.5 & 1 \\
\hline
\end{tabular}


Table 2: Blogger and non-blogger views about blogging, attitudes, norms, perceived control and intentions to blog

\begin{tabular}{lllll}
\hline & Whole sample & Bloggers & Non-bloggers & Mann \\
& Median (IQR) & Median (IQR) & Median (IQR) & Whitney p- \\
& & & & value \\
\hline Attitude & $4(0.5)$ & $4(.05)$ & $4(0.5)$ & $p=.887$ \\
Subjective norm & $5(2)$ & $5(2.5)$ & $5(3)$ & $p=.096$ \\
Perceived control & $5(3.8)$ & $6(2.5)$ & $2(4)$ & $p<.001$ \\
Intentions & $4.5(5)$ & $6(3.0)$ & $2(1)$ & $p<.001$ \\
Blogging views & $43(11)$ & $46(10.5)$ & $38(15)$ & $p<.001$ \\
\hline
\end{tabular}


Table 3: Views about blogging, attitudes, norms, perceived control and intentions to blog compared between participant occupation groups

\begin{tabular}{llllll}
\hline & Practitioners & Academics & Postgraduates & Undergraduates & Kruskall \\
& Median (IQR) & Median & Median & Median (IQR) & Wallis $p$ \\
& & $(\mathrm{IQR})$ & $(\mathrm{IQR})$ & & value \\
\hline Attitude & $4(0.5)$ & $4(0.5)$ & $4(0.5)$ & $4(0.13)$ & $p=.925$ \\
Subjective norm & $4(1)$ & $5(2)$ & $6(1.5)$ & $5.5(2.3)$ & $p=.112$ \\
Perceived & $2(6)$ & $6(3)$ & $5(3)$ & $5(3.3)$ & $p=.227$ \\
control & & & & & \\
Intentions & $1(4)$ & $6(4)$ & $4.5(4)$ & $4.5(6)$ & $p=.100$ \\
Blogging views & $38(20)$ & $43(13.5)$ & $44(9.5)$ & $46.5(10.5)$ & $p=.320$ \\
\hline
\end{tabular}


Table 4 Frequency and percentage of blog posts identified under each code with content analysis of 140 blog posts

\begin{tabular}{ccc}
\hline Code & Frequency & Percentage \\
\hline Research & 43 & $30.7 \%$ \\
Advice & 17 & $12.1 \%$ \\
Public engagement & 10 & $7.1 \%$ \\
Teaching & 14 & $10 \%$ \\
Response to event & 9 & $6.4 \%$ \\
PhD journeys & 6 & $4.2 \%$ \\
Conference reviews & 5 & $3.5 \%$ \\
Other (non-psychology) & 36 & $25.7 \%$ \\
Total & 140 & $100 \%$ \\
\hline
\end{tabular}

Note: Rounding $=99.7 \%$ 


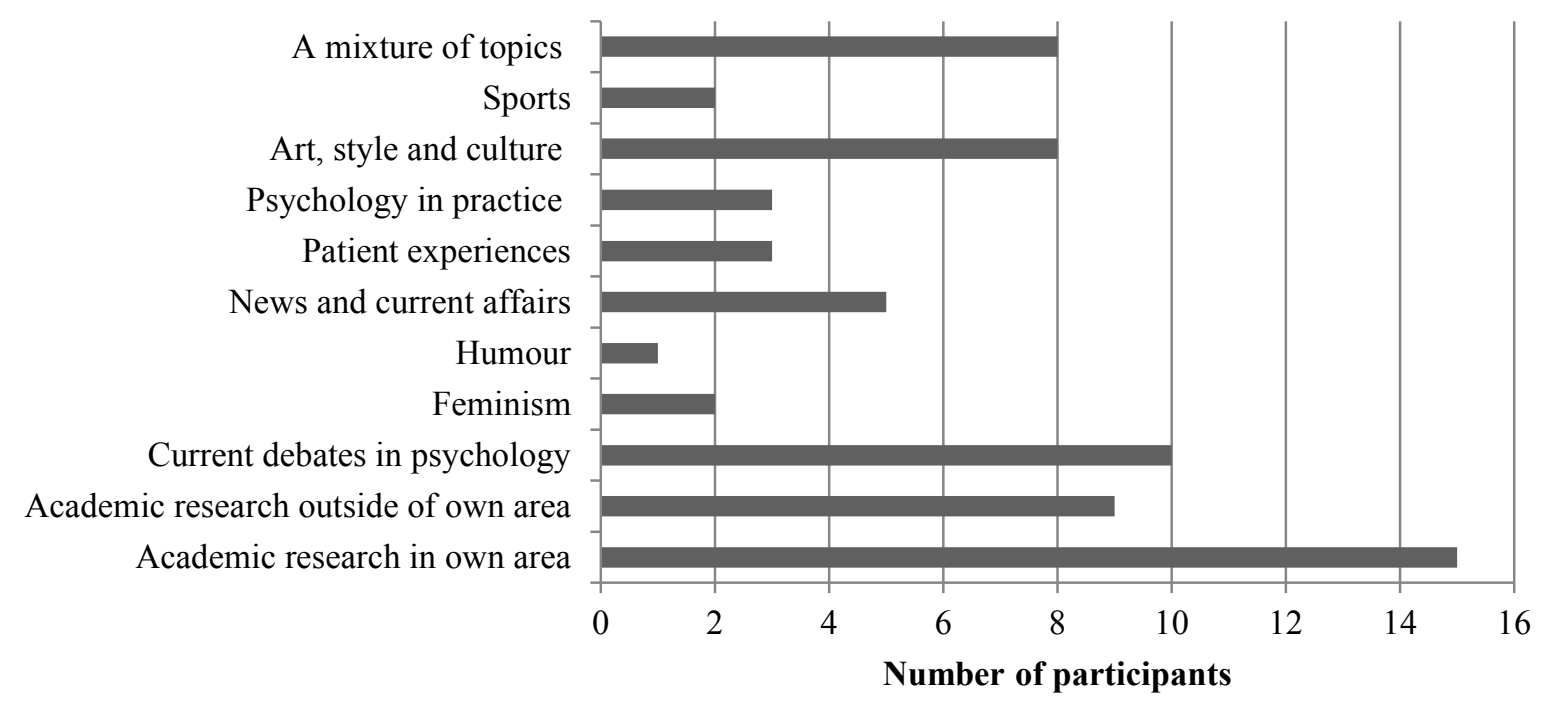

Figure 1: The content of the blogs read reported by survey participants 


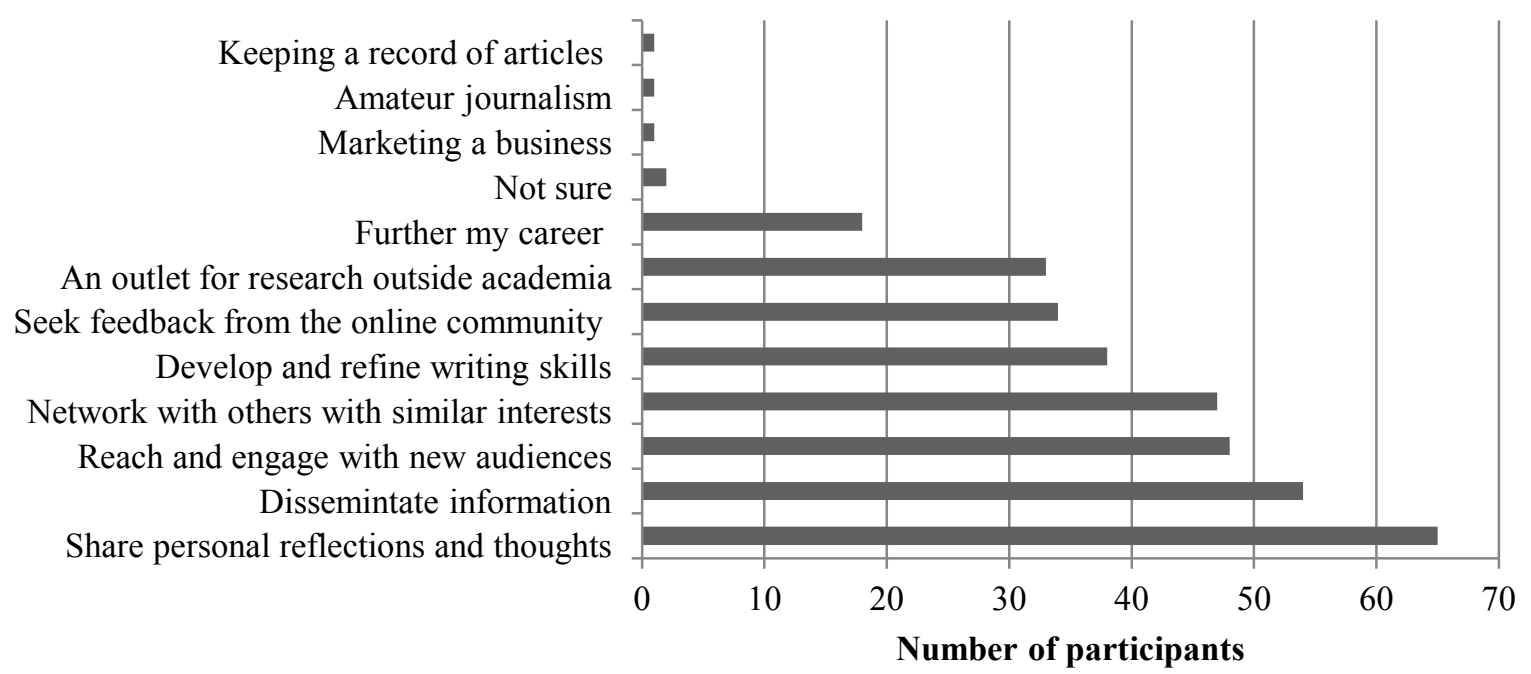

Figure 2: The purpose of blog writing reported by survey participants 